\title{
Análisis genético en pacientes con cáncer colorrectal
}

\author{
Yenny Montenegro $M^{1, a}$, José Luis Ramírez-Castro ${ }^{1}$, \\ Luis Fernando Isaza J1,2, G abriel Bedoya $B^{3, b}$, \\ Carlos Mario Muñetón-Peña ${ }^{1, b}$.

\section{Microsatellite instability among patients with colorectal cancer}

Background: In patients with colorectal carcinoma, insertions or deletions of short sequences of DNA, a phenomenon called microsatellite instability, are observed. Aim: To look for microsatellite instability and mutations of MLH1 and MSH2 gene mutations in patients with colorectal carcinoma. Material and Methods: Ten patients with sporadic colorectal carcinoma and 31 patients fulfilling criteria for hereditary nonpolyposis colon cancer (HNPCC), aged 9 to 70 years, were studied. Microsatellite instability was studied in samples of tumor and peripheral blood mononuclear cell DNA. Six markers were amplified by polymerase chain reaction and capillary electrophoresis. In samples with microsatellite instability, mutations of MLH1 and MSH2 genes were studied by direct sequencing. Results: Thirty four percent of patients had microsatellite instability and among these, $76 \%$ had a high degree of instability. BAT40 marker had the higher frequency of instability. No mutations for MLH1 and MSH2 genes were observed. However a new polymorphism, C399T, was identified in exon 3 of MSH2 gene. This polymorphism was observed both in patients with sporadic colorectal carcinoma and patients with HNPCC. Conclusions: There is a high frequency of microsatellite instability among patients with colorectal cancer. A new polymorphism, not previously reported, was identified in MSH2 gene (Rev Méd Chile 2006; 134: 1221-29).

(Key words: Colorectal neoplasms; DNA, satellite; Microsatellite repeats)

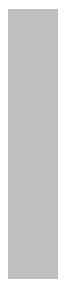

Recibido el 12 de julio, 2005. aceptado el 18 de abril, 2006.

Trabajo financiado por la Universidad de Antioquia, proyectos CODI-CPT-0018 y 0211.

${ }^{1}$ Unidad de Genética Médica, Facultad de Medicina, Universidad de Antioquia, Medellín, Colombia. ${ }^{2}$ Departamento de Cirugía, Facultad de Medicina, Universidad de Antioquia y Hospital Universitario San Vicente de Paúl, Medellín, Colombia. ${ }^{3}$ Grupo de Genética Molecular, Universidad de Antioquia, Medellín, Colombia.

aBacterióloga. Magíster en Ciencias Básicas Biomédicas.

biólogo. Magíster en Genética.

Correspondencia a: Carlos Mario Muñetón P. Unidad de Genética Médica, Facultad de Medicina, Universidad de Antioquia, Medellín, Colombia. Carrera 51D № 62-29. Fax: 2106932. Teléfono: 2106063. E mail: cmuneton@quimbaya.udea.edu.cocmuneton@gmail.com 
$\mathrm{E}^{1}$ cáncer colorrectal (CCR), a escala mundial es, en frecuencia, la tercera neoplasia y la segunda causa de muerte por cáncer en hombres y mujeres ${ }^{1}$. Según cifras mundiales, el CCR tiene una frecuencia geográfica muy variable y representa $9,4 \%$ de todos los cánceres en hombres y $10,1 \%$ en mujeres ${ }^{1,2}$.

En la población general, cerca de $80 \%$ de los casos de CCR ocurre en forma esporádica y en el resto existen antecedentes familiares, lo que sugiere un componente genético ${ }^{3}$. Del total de casos de CCR, 5 a 10\% muestra un patrón de herencia hereditario. En el CCR hereditario, se destacan principalmente el cáncer colorrectal hereditario sin poliposis (HNPCC), o síndrome de Lynch, y la poliposis adenomatosa colónica familiar (FAP), los cuales etiológicamente presentan una gran heterogeneidad genética ${ }^{4,5}$.

En especial, el HNPCC es el tipo de CCR hereditario más común, se caracteriza por un patrón de herencia autosómico dominante, con penetrancia incompleta. En la mayoría de los casos, se diagnostica antes de los 45 años y en estados avanzados de la tumorogénesis ${ }^{6}$. Además, tiene un mayor riesgo de desarrollar otros tipos de cánceres extracolónicos, como endometrio, ovario y estómago. EL HNPCC se asocia con mutaciones en varios genes del sistema de reparación de bases mal apareadas o mismatch (MLH1,MSH2, MSH6, PMS1 y PMS2). Cenca de $80 \%$ de las mutaciones informadas ocurre en los genes MLH1 y MSH26-8.

Se ha encontrado que en individuos con CCR ocurren inserciones o pérdida de secuencias repetidas cortas en el ADN (microsatélites), este fenómeno se conoce como inestabilidad microsatelital (MSI) ${ }^{9,10}$. La MSI es responsable de la acumulación de mutaciones en diferentes genes asociados con cáncer y que son cruciales en la iniciación y progresión del tumor.

El propósito de este trabajo fue realizar un estudio genético, específicamente un análisis de inestabilidad microsatelital en 41 pacientes con cáncer colorrectal y, en los casos en que se obtuvo MSI positiva (MSI+), realizar un análisis de mutaciones por secuenciamiento directo en los genes MLH1 y MSH2.

\section{PACIENTES y MÉTOdO}

Muestra. Durante el periodo comprendido entre 2000 y 2002, se estudiaron 41 pacientes con CCR, teniendo en cuenta los siguientes criterios: individuos menores de 45 años con diagnóstico clínico e histopatológico de CCR; casos de CCR con antecedentes familiares y pacientes con sospecha de HNPCC que cumplen con los criterios de Bethesda (incluyen criterios de Amsterdam) ${ }^{11}$ (Tabla 1). El promedio de edad fue de 35,9 años, con un rango entre 9 y 70 años. Los pacientes procedían de diferentes instituciones hospitalarias de Colombia, principalmente de las ciudades de Medellín (CI), Bogotá (CII), Neiva (CIII) y Santander (CIV). Este estudio fue revisado y aprobado por el Comité de Bioética de la Universidad de Antioquia. Previo consentimiento informado, se tomaron muestras de sangre periférica y del tejido tumoral a partir de biopsias frescas del tejido afectado y de bloques de parafina de los archivos de los diferentes departamentos de patología de hospitales que colaboraron con este estudio. Los diagnósticos se confirmaron mediante revisión de las historias clínicas y exámenes de patología.

Extracción de ADN. Se aisló el ADN a partir de linfocitos de sangre periférica empleando el kit comercial Wizard de Promega, siguiendo las instrucciones del fabricante. El ADN del tejido tumoral (biopsia fresca y microdisección a partir de bloques de parafina), se obtuvo con el método convencional de fenol-cloroformo y precipitación con etanol frío.

Análisis de MSI. Se realizaron a partir de muestras de ADN de sangre y del tejido tumoral de cada paciente. Para determinar MSI se utilizaron los 6 marcadores microsatelitales recomendados internacionalmente por el National Cancer Institute Worshop (también conocidos como Bethesda panel) ${ }^{12}$ : BAT-25, BAT-26, BAT-40, D17S250, D2S123 y D5S346. Estos microsatélites se amplificaron utilizando los cebadores que aparecen en la Tabla 2, marcados con Cy5 (Pharmacia Biotech), bajo las condiciones de reacción previamente informadas $^{13}$. Los tamaños alélicos se resolvieron en una electroforesis en geles de poliacrilamida al 8\%, utilizando un analizador genético ALFexpress 2.0 (Pharmacia Biotech) y empleando los programas 
Tabla 1. C riterios clínicos establecidos por consenso para el diagnóstico de individuos con H N PC C 11

\begin{tabular}{|ll|}
\hline Nombre & Criterios \\
\hline Amsterdam modificados & 1.
\end{tabular}

Tabla 2. Juegos de cebadores empleados para la amplificación de los 6 marcadores utilizados para la evaluación de M SI, en los pacientes con C CR estudiados

\begin{tabular}{|lll|}
\hline STR & Forward & Reverse \\
\hline BAT-25 & TCGCCTCCAGAATGTAAGT & GAGCCATAGTTAAAATGCAGA \\
BAT-26 & TGACTACTTTTGACTTCAGCC & GGGTTAAAAATGTTGATTGGTT \\
BAT-40 & ATTAACTTCCTACACCACAAC & CAAGGTGGTCTTGCTCTAC \\
D2S123 & CAGGATGCCTGCCTTTA & GTCCCATAGGTGGAAAGTCC \\
D5S346 & ACTCACTCTAGTGATAAATCGGG & CTAGTAATACTGTCTTATCTGCT \\
D17S250 & GGAAGAATCAAATAGACAAT & GGTTTAAATATATATATGGCCAGC \\
\hline
\end{tabular}

ALFwin y Alelelinks. Se definió MSI por la presencia de nuevas bandas en la muestra del ADN tumoral (las cuales podrían presentar pérdida o ganancia de alelos), luego de la amplificación por PCR, que no están en el amplicón del $\mathrm{ADN}$ normal. Se siguieron los criterios internacionales del NCI para la determinación de MSI en
CCR y las muestras se clasificaron sobre la base de una alta o baja frecuencia de inestabilidad ${ }^{12}$. Así, MSI alta (MSI-H), si dos o más de los 6 marcadores en el tumor muestran inestabilidad (inserción/ deleción); MSI baja (MSI-L) si sólo un marcador de los 6 es inestable; y estables (MSS) si no se detecta inestabilidad en los 6 marcadores. 
Análisis de mutaciones. Se realizaron en individuos con MSI positiva (MSI+). Se amplificaron los exones 1, 8, 13, 14 del gen MLH1 y 3, 6 y 12 del gen MSH2, tanto en el ADN del tumor como en el del tejido normal, en los cuales se han informado previamente mutaciones para la población hispanoamericana ${ }^{14}$. Los cebadores utilizados se muestran en la Tabla 3, (Integrated DNA technologies, INC. USA). Las condiciones de las reacciones de amplificación se siguieron con las informadas previamente $^{15}$. Los amplicones obtenidos se purificaron con el kit Wizard DNA Purification (Promega), siguiendo las instrucciones del fabricante. Para el secuenciamiento se utilizó el Terminador Sequencing Kit y las reacciones de secuencia se resolvieron mediante electroforesis capilar en un analizador genético ABI 310 (Applied Biosystems, CA) empleando el programa Navegator.

\section{Resultados}

Se estudió en total 41 pacientes colombianos con CCR (la mayoría procedentes de las ciudades de Bogotá, Medellín y Neiva). De éstos, 39,5\% fueron mujeres y $60,5 \%$ hombres; el promedio de edad fue de 35,9 años con un rango entre 9 y 70 años; $74,4 \%$ de los pacientes era menor de 40 años y 25,6\% mayor de 41 años.

Según los criterios internacionales establecidos para la identificación de pacientes con HNPCC, se encontró que $31(75,6 \%)$ cumplían los criterios de Bethesda. Entre las familias estudiadas, se identificó una con síndrome de Lynch tipo I (grupos familiares que cumplen los criterios de Amsterdam y en las cuales solamente se presenta CCR) y tres familias con síndrome de Lynch tipo II (agrega- ción familiar de diferentes tumores en familias que cumplen los criterios de Amsterdam). Cuarenta y nueve por ciento de los casos presentaron antecedentes familiares de algún tipo de cáncer, mientras que $51,2 \%$ restante no tenían antecedentes.

El 88,1\% (37/41) de los CCR estudiados se localizaron en la región ascendente del colon (24), seguido por colon transverso (9) y colon descendente (4). Los cuatro restantes se localizaron en el recto.

En la Tabla 3 se presentan los resultados sobre la variedad histológica y el grado de los CCR estudiados. Se clasificaron en cinco grupos. El más frecuente fue el adenocarcinoma bien diferenciado, presente en $71,4 \%$ de los casos.

Con respecto a la clasificación TNM de las muestras, los tumores se clasificaron de la siguiente forma: T1SN0M0 (4,7\%), T2N0M0 (19,1\%), T3N2M0 y T2N1M0 $(47,6 \%)$ y T3N2M1 $(28,6 \%)$.

Inestabilidad microsatelital. Con los 6 marcadores utilizados, se determinó MSI positiva en 14 de los 41 pacientes analizados (34,1\%). La MSI+ observada estuvo presente en uno o en más de un marcador utilizado. Este resultado está en el rango de MSI informado en individuos con CCR. De los 14 pacientes con MSI+, 11 (26,8\%) presentaron MSI alta (MSI-H), es decir, pacientes con una alta probabilidad de tener HNPCC y tres $(7,3 \%)$ con MSI baja (MSI-L). El promedio de edad de los individuos con MSI fue de 39,3 años (con un rango entre 29 y 59 años).

En la Tabla 4 se describe la relación entre los resultados obtenidos de MSI y el lugar de procedencia de los pacientes. Se encontró MSI-H en individuos procedentes de las cuatro ciudades (I-V), siendo las ciudades CI y CIII las que presentaron un mayor número de individuos con inestabilidad.

Tabla 3. D istribución de la variedad y grado histológico encontrados en los 41 casos de CCR examinados

\begin{tabular}{|lcc|}
\hline Variedad histológica & № casos & $\%$ \\
\hline Adenocarcinoma bien diferenciado & 30 & 71,4 \\
Adenocarcinoma moderadamente diferenciado & 2 & 4,8 \\
Adenocarcinoma mal diferenciado & 4 & 9,5 \\
Adenocarcinoma mucinoso & 4 & 9,5 \\
Tumor de células en anillo & 2 & 4,8 \\
Total & 41 & 100 \\
\hline
\end{tabular}


Tabla 4. D istribución por ciudades del tipo de M SI detectada en 14 individuos con CCR. $\mathrm{N}$ ótese que en todas las ciudades se identificaron individuos con $\mathrm{M} \mathrm{SI-H}$

\begin{tabular}{|lccc|}
\hline Ciudades & MSI-H & MSI-L & Total \\
\hline Medellín (CI) & 3 & 2 & 5 \\
Bogotá (CII) & 2 & 0 & 2 \\
Neiva (CIII) & 4 & 1 & 5 \\
Santander (CIV) & 2 & 0 & 2 \\
Total & 11 & 3 & 14 \\
\hline
\end{tabular}

C: Ciudades. CI: Medellín (noroccidente del país); CII: Bogotá (centro) CIII: Neiva (suroccidente) CIV: Santander (nororiente). MSI-H: inestabilidad alta. MSI-L: inestabilidad baja.

El microsatélite con mayor inestabilidad fue BAT-40, seguido por D2S123; el de menor inestabilidad fue el marcador D5S346. En este estudio se identificaron los siguientes tipos de MSI en el ADN tumoral, pérdida en el tamaño de los alelos (60\%) (Figura 1), inserciones (22,5\%) y múltiples alelos $(12,5 \%)$. Además, se detectó pérdida de heterocigocidad ( $\mathrm{LOH}$ ) en $5 \%$.

Mutaciones en los genes MLH1 y MSH2. Estudios de secuenciamiento se realizaron solamente en pacientes con MSI-H, no se detectaron mutaciones en los exones evaluados de los genes MLH1 y MSH2. Se detectó un nuevo polimorfismo, una transición de $\mathrm{C}$ por $\mathrm{T}$ en el exón 3 del gen MSH2 sobre el nucleótido 399 (C399T) en la tercera posición del codón 133 (GAT/GAC) (Figura 2), que codifica para el ácido aspártico; este cambio no implica variación en el aminoácido codificado $\mathrm{y}$, por lo tanto, no cambia la secuencia de la proteína. Este cambio puede considerarse como un polimorfismo, puesto que se encontró tanto en pacientes con CCR esporádico y HNPCC, como en individuos sanos.

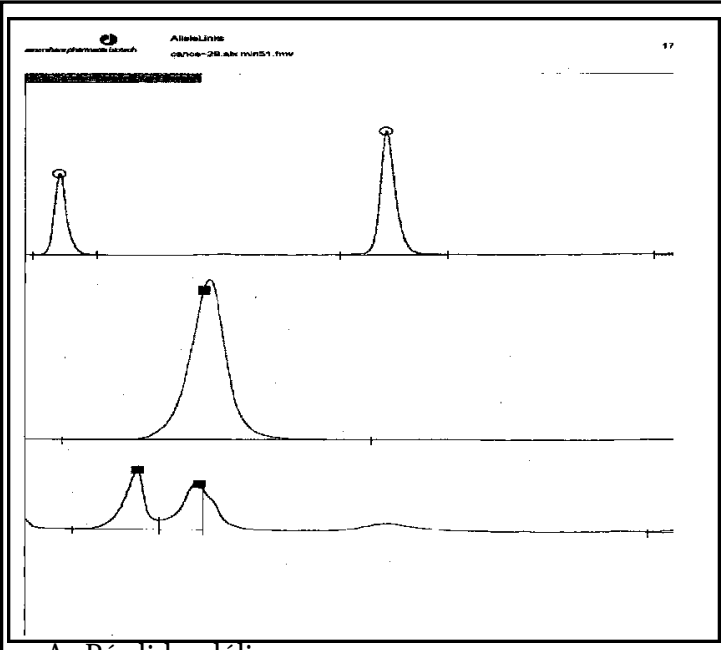

A. Pérdida alélica

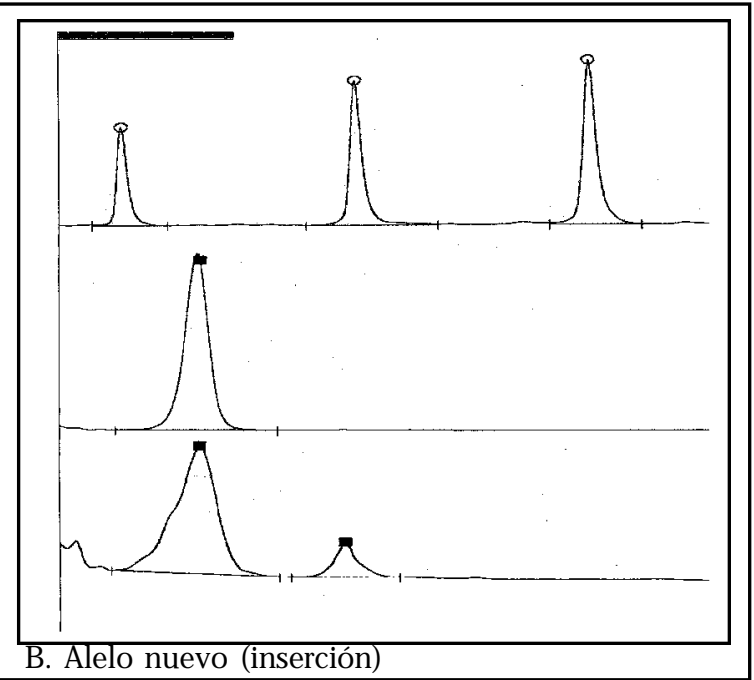

Figura 1. Detección de inestabilidad microsatelital utilizando los 6 marcadores recomendados mediante un secuenciador automático. A. Se observa la pérdida o disminución del tamaño alélico en el ADN tumoral de un individuo con CCR y MSI positiva. B. Se muestra la aparición de un alelo nuevo en el ADN tumoral en uno de los pacientes estudiados. En el centro: ADN tejido normal. Línea inferior: ADN del tejido tumoral. 


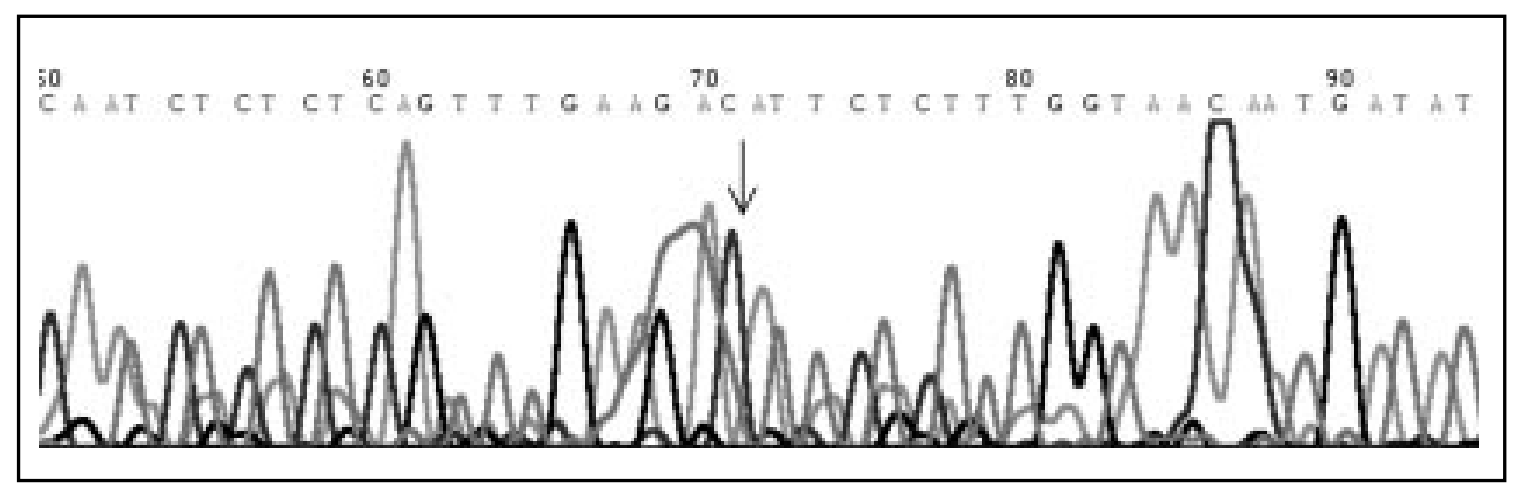

Figura 2. Secuencia parcial de la región del gen MSH2, que muestra el polimorfismo nuevo C399T identificado en el exón 3, en la tercera posición del codón 133, en pacientes con CCR que presentaron MSI-H.

\section{DisCUSIÓN}

Se realizó un estudio clínico-patológico y molecular del CCR esporádico y de tipo hereditario (HNPCC), según los criterios de Bethesda. En su etiología, el CCR presenta una gran heterogeneidad genética, además de la interacción con factores ambientales. Existen pocos trabajos en poblaciones hispanoamericanas sobre los aspectos genéticos de este tipo de cáncer, especialmente en lo referente a la inestabilidad microsatelital.

En la muestra analizada, la frecuencia de CCR fue 1,5 veces mayor en hombres que en mujeres, esta cifra es similar a las informadas en algunos estudios realizados en poblaciones hispanoamericanas, en las cuales se ha observado un aumento de CCR en los hombres ${ }^{2}$. Cabe mencionar que previamente informamos en dos poblaciones colombianas con CCR una frecuencia cercana a $20 \%$ en individuos menores de 40 años $^{16}$.

En este estudio se identificaron 11 pacientes menores de 25 años, entre ellos, cuatro menores de edad, cifra significativa si se tiene en cuenta que existen pocos estudios con CCR en este grupo etáreo; este resultado podría sugerir un efecto de anticipación en nuestra población por efectos de heredabilidad (agregación familiar) y de factores medioambientales sobre la expresión del CCR.

Según la localización y estadio del CCR, la muestra estudiada tuvo el mismo comportamiento que esta entidad ha mostrado en otras poblaciones, siendo más frecuente el tumor en el colon ascen- dente $^{17,18}$. Además, cabe mencionar que, al momento del estudio, la mayoría de los individuos presentaban un estado avanzado del cáncer, es decir, con metástasis en varios ganglios linfáticos 0 con metástasis a distancia y, por tanto, un mal pronóstico de la enfermedad. Lo anterior llama la atención, puesto que un alto número de estos individuos (74,4\%) eran menores de 40 años.

También se pretendía identificar, entre todos los pacientes con CCR de este estudio, individuos con sospecha de HNPCC, según los criterios de Bethesda, dada la flexibilidad de estos criterios para la identificación de individuos con HNPCC, comparada con los criterios de Amsterdam que son más rigurosos. Se encontró que $75,6 \%$ de la muestra seleccionada cumplió los criterios de Bethesda. Estos criterios se consideran muy sensibles, aunque poco específicos para seleccionar pacientes sospechosos con HNPCC; sin embargo, otros autores proponen que los criterios de Bethesda podrían ser el criterio clínico con mayor sensibilidad y especificidad para la identificación de individuos con mutaciones en los genes MLH1 y MSH2, además de presentar mayor facilidad en su uso para la práctica clínica $^{19}$.

Con respecto a los microsatélites, los más inestables fueron BAT-40, D2S123 y BAT-26, similar a lo informado para pacientes con HNPCC ${ }^{20-22}$. Sin embargo, algunos trabajos en población finlandesa muestran que BAT-26 es uno de los marcadores mas inestables en familias con HNPCC $^{19,23}$; en este estudio, BAT26 fue el tercer marcador más inestable. 
Con los resultados obtenidos, podría concluirse que la MSI es un evento común en el CCR y que sólo con los marcadores BAT-40, D2S123 y BAT-26, se podría evaluar e identificar todos los pacientes que presentaron MSI+ y no utilizar 6 marcadores, en la misma forma como lo plantearon Ward et al, para la población australiana ${ }^{20}$. Sin embargo, en nuestro caso sería necesario aumentar el tamaño de la muestra poblacional y luego evaluar la posibilidad de disminuir el número de marcadores.

Por otra parte, las pruebas de MSI se han utilizado para ayudar a determinar la probabilidad de que un individuo con CCR presente HNPCC ${ }^{10}$. Por tal razón se desarrollaron los criterios de Bethesda con el objeto de establecer una guía para el diagnóstico de HNPCC ${ }^{11,23}$. De esta mane$\mathrm{ra}$, individuos con MSI-H podrían tener una mayor probabilidad de tener mutaciones en los genes de reparación MLH1 y MSH2 24 .

De acuerdo con lo anterior, los 11 (26,8\%) individuos que presentaron MSI-H pueden considerarse con diagnóstico de HNPCC; además, cumplían con los criterios de Bethesda. Otros estudios han encontrado hasta $90 \%$ de MSI-H en CCR asociado con HNPCC ${ }^{10,25,26}$. Sin embargo, los resultados obtenidos de MSI-H y MSI-L están entre los rangos publicados en la literatura ${ }^{20-22}$. En cuanto a los individuos con MSI-L, éstos podrían excluirse como HNPCC positivos. Varios trabajos proponen que los casos de CCR con MSI$\mathrm{L}$ se deben a vías genéticas diferentes que se presentan en el desarrollo de este tipo de cáncer $^{19,20,23}$. En síntesis, la MSI, más los antecedentes familiares y una rigurosa evaluación clínica basada en los criterios de Bethesda, son de gran importancia para el diagnóstico e identificación de pacientes HNPCC.

Debe tenerse en cuenta que entre 15 y $20 \%$ de los CCR esporádicos presentan MSI-H, en su mayoría originada por hipermetilación en el promotor del gen MLH16,27,28. En este estudio, 51,2\% de los casos esporádicos no mostraron MSI, por tanto se podría descartar la hipermetilación de este gen; sin embargo, este mecanismo epigenético podría estar presente en otros genes relacionados con el CCR, como el gen k-ras, que se encuentra alterado hasta en $50 \%$ de los casos ${ }^{29}$.

Los estudios de MSI se consideran como una buena estrategia para iniciar el tamizaje molecular de familias con sospecha de HNPCC, antes de realizar el secuenciamiento completo de los genes MLH1 y MSH2, especialmente cuando se consideran los costos de este último. En los casos de HNPCC (con MSI$\mathrm{H}$ ) identificados, no se detectaron mutaciones en los siete exones secuenciados para los genes MLH1 y MSH2. Se sugiere secuenciar los exones restantes de estos genes, con miras a determinar la presencia de mutaciones en estos pacientes como la causa molecular. Podnía también mencionarse que la no detección de mutaciones podnía deberse al tamaño de la muestra, puesto que se ha calculado que la frecuencia de mutación germinal en pacientes con HNPCC oscila entre 0,9 y $2,7 \% \%^{6}$. Asimismo, no se debe descartar mutaciones en otros genes del sistema MMR (como el MSH3, MSH6 o PMS1) ${ }^{6,22,30}$. Resultados similares se han obtenido en otros trabajos en los cuales se encuentran casos con MSI positiva y ausencia de mutaciones en los genes MLH1 y MSH2 ${ }^{10,21,31}$. En tal situación, podnían considerarse otras explicaciones, además de las ya mencionadas, como alteraciones en otros genes relacionados con CCR (k-ras, p53, TGFßRII o BAX), fenómenos de hipermetilación que afectan la expresión de los genes involucrados o a reordenamientos genómicos (grandes deleciones), que se presentan con mayor frecuencia en el gen MSH2, los cuales no son detectados con las técnicas convencionales de análisis de mutaciones ${ }^{6,8,32-34}$. Por tal razón, muchos casos podńan quedar inconclusos desde el punto de vista molecular.

Finalmente, en este estudio informamos la identificación de un nuevo polimorfismo C399T en el gen MSH2 en individuos con CCR esporádico y HNPCC en una muestra de la población colombiana. Este polimorfismo, aunque se encuentra anotado en una de las bases de datos para HNPCC $^{35}$, no se ha informado en la literatura impresa, por lo tanto, se informa por primera vez en la población hispanoamericana. El significado de este polimorfismo en CCR aún no está bien conocido. Podría sugerirse que esta variación hace parte del componente genético de la población colombiana, la cual es históricamente aislada y, por tal motivo, podría estar asociada con la etiología del HNPCC, tal como se ha podido establecer para otros tipos de enfermedades genéticas en nuestra población ${ }^{36,37}$. Además, el haber identificado MSI-H en individuos de diferentes regiones del país y a la ya conocida alta frecuencia de CCR en individuos jóvenes colombianos ${ }^{16}$, 
podría proponerse que una alteración genética, más los factores ambientales de cada región, estarían incidiendo para que se presente una mayor susceptibilidad para desarrollar CCR. Sin embargo, son necesarios estudios adicionales para

\section{REFERENCIAS}

1. Jemal A, Thomas A, Murray T, Thun M. Cancer statistics 2002. CA Cancer J Clin 2002; 52: 181-2.

2. Boyle P, Langman JS. ABC of Colorectal Cancer. Epidemiology. BMJ 2000; 321: 805-8.

3. Calvert PM, Frucht H. The Genetics of Colorectal Cancer. Ann Intern Med 2002; 137: 603-12.

4. Lynch HT, De La Chapele A. Genetic susceptibility to non-polyposis colorectal cancer. J Med Genet 1999; 36: 801-18.

5. Montenegro Y, Ramírez A, Muñetón C. Cancer colorectal heredado. Rev Colomb Cir 2002; 17: 30-6.

6. Lynch T, De La Chapele A. Hereditary colorectal cancer. N Engl J Med 2003; 348: 919-32.

7. Peltomaki P, Vasen HF. Mutations predisposing to hereditary nonpolyposis colorectal cancer: database and results of a collaborative study. The Internatinal Collaborative Group on Hereditary Nonpolyposis Coleractal Cancer. Gastroenterology 1997; 113: 1146-58.

8. Wheier JM, MoRTensen NJ. DNA mismatch repair genes and colorectal cancer. Gut 2000; 47: 148-53.

9. Aaltonen L, Peitomaki P, Meckin J, Jarvinen H, Jass JR, GREEN JS ET AL Replication emors in benign and malignant tumors from hereditary nonpoliposis colorectal cancer patients. Cancer Res 1994; 54: 1645-8.

10. Vilkki S, Launonen V, Karhu A, Sistonen P, Västrik I, AaLtonen LA. Screening for microsatellite instability target genes in colorectal cancers. J Med Genet 2002; 39: 785-9.

11. Rodríguez-Bigas MA, Boland CR, Hamilton SR, Henson DE, Jass JR, Khan PM et al. A National Cancer Institute Workshop on Hereditary Nonpolyposis Colorectal Cancer Syndrome: meeting highlights and Bethesda Guidelines. J Natl Cancer Inst 1997; 89: 1758-62.

12. Boland CR, Thibodeau SN, Hamliton SR, Sidransky D, Eshleman R, BuRT RW ET AL. A National Cancer Institute Workshop on Microsatellite Instability for cancer detection and familial predisposition: la caracterización molecular en la población estudiada, como también determinar el papel de este polimorfismo en la etiología del CCR en la población hispanoamericana en general.

development of international criteria for the determination of microsatellite instability in colorectal cancer. Cancer Res 1998; 58: 5248-57.

13. Gryfe R, Kim H, Hsieh ETK, Aronson MD, Holowaty EJ, BuL SB ET aL. Tumor Microsatellite Instability and Clinical Outcome in young patients with Colorectal Cancer. N Engl J Med 2000; 342(13): 69-77.

14. Godino J, De La Hoya M, Diaz-Rubio E, Bentio M, CALÉS T. Novel Germline MLH1 and MSH2 Mutations in Hereditary Non-Polyposis Colorectal Cancer Families from Spain. Human Mutation 2001; 467: 2-6.

15. Wijnen J, Vasen H, Meera KP, Menko FH, Van DeR Kuft H, Van Leeuwen C et al. Seven new mutations in MSH2 an NPCC gene, identified by denaturing gradient gel electrophoresis. Am J Hum Genet 1995; 56: 1060-6.

16. Montenegro Y, Ramírez A, Muñetón C, Isaza L, Ramírez J. Comportamiento del Cáncer colorrectal en pacientes menores de cuarenta años del hospital universitario Hernando Moncaleano Perdomo de Neiva (HUHMP) y el Hospital Universitario San Vicente de Paúl de Medellín (HUSVP) entre 1980 y 2000. Rev Colomb Cir 2002; 17: 10-4.

17. American Gastroenterology Association. Molecular diagnosis of Cancer predisposition. Biochemical \& Biophysica 1999; 1923: 1-10.

18. Curran B, Lenehan K, Mulcahy H, Tighe O, Bennett MA, KAY EW ET AL. Replication errors phenotype, clinico pathological variables, and patient outcome in Dukes B stage II (T3, NO, MO) colorectal cancer. Gut 2000; 46: 200-4.

19. Syngal S, Fox EA, Eng C, Kolodner RD, Garber JE. Sensitivity and specificity of clinical criteria for hereditary non-polyposis colorectal cancer associated mutations in MSH2 and MLH1. J Med Genet 2000; 37: 641-5.

20. Ward R, Meagher A, TOMLinson I, O'Connor T, NoRRIE M, Wu R ET AL. Microsatellite Instability and the clinicopayhological features of sporadic colorectal cancer. Gut 2001; 48: 821-9. 
21. Tang R, Rong C, Chim Wu M, Fan CW, Lu KW, CHEN JS ET AL. Colorectal cancer without high microsatellite instability and chromosoma Instability- an alternative genetic pathway to human colorectal cancer. Carcinogenesis 2004; 25: 841-6.

22. Loukola A, Ekin K, Laiho P, Salovaara R, Kristo P, JaRVINEN H ET AL. Microsatellite Marker Analysis in Screening for Hereditary Nonpolisis Colorectal Cancer (HNPCC). Cancer Res 2001; (6): 4545-9.

23. Morifuji M, Hiyama E, Murakami $Y$, Imamura $Y$, Sueda T, Үоконама T. Fluorescent-based BAT-26 analysis for distinct screening of microsatellite instability in colorectal cancers. Int J Oncol 2003; 22: 807-13.

24. Jeong SY, Shin KH, Shin JH, Ku JL, Shin YK, ParK SY ET AL. Microsatellite instability and mutations in DNA mismatch repair genes in sporadic colorectal cancers. Dis Colon Rectum 2003; 46: 1069-77.

25. Nash GM, Gimbel M, Shia J, Cu山ford AT, Nathanson DR, NdubuisI M ET AL. Automated, multiplex assay for high-frequency microsatellite instability in colorectal cancer. J Clin Oncol 2003; 21: 3105-12.

26. Rigau V, Sebbagh N, Olschwang S, Paraf F, Mourra N, PARC Y ET AL. Microsatellite instability in colorectal carcinoma. The companison of immunohistochemistry and molecular biology suggests a role for hMSH6 immunostaining. Arch Pathol Lab Med 2003; 127: 694-700.

27. TOMLiNson I. Different pathways of colorectal carcinogenesis and their clinical pictures. Annals NY Acad Science 2000; 9: 10-20.

28. Cunningham JM, Christensen ER, Tester DJ, Kim CY, Roche PC, BuRgart LJ et al. Hypermetilation of the hMLH1 promoter in colon cancer with microsatellite instability. Cancer Res 1998; 58: 3455-6.

29. Esteler M, Toyota M, Sánchez-Céspedes M, Capela G, Peinado MA, Watkins DN et al. Inactivation of DNA RepairGene 06 Metylguanine - DNA Methyltransferase by Promoter hypermethylation in Asso- ciated with G to A Mutations in K-Ras in Colorectal tumorigenesis. Can Res 2000; 60: 2368-71.

30. Wagner A, Tops C, Wijnen JT, Zwinderman K, Van Der Meer C, Kets M et aL. Genetic testing in hereditary non-polyposis colorectal cancer families with a MSH2, MLH1, or MSH6mutation. J Med Genet 2002; 39: 833-7.

31. Di Fiore F, Charbonnier F, Martin C, Frerot S, Olschwang S, Wang Q ET al. Screening for genomic rearrangements of the MMR genes must be included in the routine diagnosis of HNPCC. J Med Genet 2004; 41: 18-20.

32. Shen L, Kondo Y, Hamilton SR, Rashid A, Issa JP. P14 methylation in human colon cancer is associated with microsatellite instability and wild-type p53. Gastroenterology 2003; 124: 626-33.

33. Hasegana A, Fukushima T, Mashiko M, Nakano K, Suzuki S, ENDO Y ET AL. Alterations of the K-ras and p53 genes and microsatellite instability in sporadic colorectal carcinomas. Anticancer Res 2004; 24: 2047-52.

34. Wagner A, Barrows A, Wijnen JT, Van Der Kuft H, FRANKen PF, VerkujJien P et AL. Molecular analysis of hereditary nonpolyposis colorectal cancer in the United States: high mutation detection rate among clinically selected families and characterization of an American founder genomic deletion of the MSH2 gene. Am J Hum Genet 2003; 72: 1088-100.

35. Intemational Collaborative Group on Hereditary Nonpolyposis Colorectal Cancer. http://www.nfdht.nl

36. Carvajal L, Soto I, Pineda N, Ortiz-Barrientos D, Duque C, Ospina-Duque J et al. Strong Amerind/White Sex Bias and a Possible Sephardic Contribution among the Founders of a Population in Nortwest Colombia. Am J Hum Genet 2000; 67: 1287-95.

37. Ramírez-Castro JL, Pineda-Trujшo N, Valencia aV, MuÑETÓN CM, BOtero O, TRUJLO O ET AL. Mutations in FOXL2 undenlying BPES (types 1 and 2) in Colombian families. Am J Med Genet 2002; 113: 47-51.

Agradecimientos

Agradecemos a los pacientes y sus familiares por su participación en este trabajo. A los cirujanos, enfermeras y a los departamentos de patología de las instituciones de salud que intervinieron en el trabajo por su valiosa colaboración (Hospital Militar Central, Bogotá; Hospital San Vicente de Paúl, Medellín; Hospital Hernando Moncaleano Perdomo, Neiva, entre otros). Este estudio fue financiado por la Universidad de Antioquia, proyectos CODI-CPT-0018 y 0211. 